\title{
Percepción docente sobre la gamificación de la evaluación en la asignatura de Historia en educación secundaria
}

\author{
Ernesto Colomo-Magaña, Enrique Sánchez-Rivas, Julio Ruiz-Palmero* y José Sánchez-Rodríguez \\ Universidad de Málaga, Málaga -España (correo-e: ecolomo@uma.es; enriquesr@uma.es; julio@uma.es; \\ josesanchez@uma.es). \\ * Autor a quien debe ser dirigida la correspondencia.
}

Recibido Mar. 6, 2020; Aceptado May. 7, 2020; Versión final May. 22, 2020, Publicado Ago. 2020

\section{Resumen}

El objetivo de este estudio es analizar la percepción de los docentes respecto a la aplicación de un examen gamificado para evaluar la asignatura de Historia en educación secundaria. La muestra la conforman 192 profesores de Geografía e Historia de la provincia de Málaga (España), separados en un grupo control (110), que aplicarán un examen tradicional, y un grupo experimental (82), que implementarán el de base lúdica. A ambos grupos se le administró un cuestionario estandarizado sobre evaluación gamificada. Los resultados reflejan que la prueba gamificada es mejor percibida por los docentes que la tradicional, encontrando diferencias significativas en todas las áreas analizadas. Considerando los resultados de este estudio, se concluye que la evaluación gamificada es mejor valorada por los docentes, destacando como principales ventajas el aumento de la motivación y la capacidad de la prueba para dar continuidad al aprendizaje fuera del contexto escolar.

Palabras clave: educación secundaria; evaluación de la educación; gamificación; enseñanza de la historia

\section{Teaching perception about gamification of the evaluation in the subject of History in secondary education}

\begin{abstract}
The objective of this study was to analyze the perception of teachers regarding the application of a gamified exam to evaluate the subject of History in secondary education. The sample was composed of 192 professors of Geography and History from the province of Malaga (Spain). They were separated into a control group (110 professors) that applied a traditional exam and an experimental group (82 professors) that implemented the gamified exam. Both groups were administered a standardized questionnaire on gamified evaluation. The results showed that the gamified exam was better perceived by teachers than the traditional exam. There were significant differences between both exams in all areas analyzed. In conclusion, the gamified evaluation was better valued by teachers than the traditional exam. Gamified evaluation was highlighted by two main advantages: 1) the increase in motivation and 2) the capacity of the exam to facilitate continuity to learning outside the school context.
\end{abstract}




\section{INTRODUCCIÓN}

Conocer nuestro pasado nos permite entender cómo hemos llegado al momento actual, pudiendo tomar conciencia crítica de cómo los sucesos y acontecimientos inciden en el devenir del contexto del ser humano. No podemos obviar que el hombre es el resultado de interactuar con el mundo y la realidad (Colomo y Domínguez, 2015), por lo que conocer cómo fueron esas relaciones y sus consecuencias nos permitirán prever hacia qué tipo de futuro nos dirigimos, evitando aquello que reporte efectos negativos a la humanidad. La asignatura de Historia nos permite entender la sociedad actual y conocer el presente desde una mirada a lo acontecido en el pasado. Para lograr desarrollar un proceso de enseñanza-aprendizaje de la Historia que sea positivo y que conceda a esta disciplina la importancia social que tiene, la evaluación se convierte en un elemento clave. Y es que el estudio de la Historia no se puede reducir a memorizar fechas, nombres y datos, sino a lograr adquirir una visión crítica histórica que fomente la reflexión y el pensamiento a la hora de actuar en sociedad (Ojeda, 2018). En este sentido, la evaluación sigue siendo el proceso por excelencia para corroborar, mediante la recogida de información de forma sistemática, la adquisición de los conocimientos y el grado de desarrollo de las competencias, con el propósito de poder tomar decisiones respecto al proceso de enseñanza-aprendizaje para su mejora. Por esta razón, el foco de este estudio se situará en cómo se evalúa la asignatura de Historia.

Pese a que son múltiples los instrumentos, metodologías y tareas que se pueden aplicar para evaluar los procesos de aprendizaje del alumnado, como portafolios, aprendizaje basado en proyectos, flipped classroom, gamificiación, etc., el examen sigue siendo la prueba más implementada para evaluar la asignatura de Geografía e Historia en Educación Secundaria (Miralles y Monteagudo, 2019). En este sentido, aunque el estudio de Álvarez (2018) pone de manifiesto la posibilidad y predisposición del profesorado a implementar procesos de enseñanza-aprendizaje que favorezcan el aprendizaje significativo y el pensamiento histórico mediante el planteamiento de un nuevo curriculum y la incorporación de las TIC, los contenidos conceptuales y factuales se siguen valorando a través de exámenes. De forma semejante ocurre en la investigación de Alfageme et al. (2015), donde el profesorado no contempla la necesidad de adecuar la forma de evaluar al desarrollo de procesos innovadores, confundiendo la evaluación continua con la realización de exámenes de forma más periódica.

Ante esta praxis, que sigue anclada en una visión tradicional y memorística del proceso educativo, donde prima la capacidad de retención sobre otras capacidades y competencias como el pensamiento crítico o la reflexión, estudios como el de Agudelo et al. (2018) creen en la necesidad de repensar el sentido de la evaluación, fomentando la diversificación de los instrumentos y técnicas para evaluar y reduciendo el protagonismo que se le sigue otorgando al examen tradicional (Trevitt et al., 2012). El motivo es la finalidad sumativa del examen, donde se concede toda la importancia al resultado en detrimento del proceso formativo, no concediendo importancia a los aprendizajes continuos que se realizan en el transcurso de la asignatura. El problema es que todas las tareas que conforman el acto educativo y el curriculum, incluida la evaluación, deben estar orientadas a promover el aprendizaje del alumnado y no tener un propósito exclusivamente valorativo, sino también integral y humanizante (Acebedo et al., 2017). Una visión pedagógica que es compartida entre los docentes de Geografía e Historia (Monteagudo et al., 2015). Por ello, este estudio pretende profundizar en la valoración de los docentes respecto a la implementación de mecanismos e instrumentos de evaluación con base lúdica.

La adaptación de acciones, tareas y propuestas de enseñanza-aprendizaje en juegos se denomina gamificación (Melo y Díaz, 2018), cuyo fin es fomentar el compromiso del alumnado respecto a un proceso formativo caracterizado por la motivación, la diversión o la superación ante los retos, en un contexto que prioriza el aprendizaje significativo y trata de evitar un clima de competitividad. Se trata de favorecer la disposición del discente hacia el aprendizaje a través de elementos didácticos con carácter lúdico (Hanus y Fox, 2015), tratándose de una propuesta que es bien recibida tanto por el propio alumnado, quienes manifiestan que jugando aprenden más y es su actividad preferida (Bicen y Kocakoyun, 2018; Riemer y Schrader, 2015); como por los docentes, quienes muestran una percepción positiva respecto a su implementación (González et al., 2019) por su carácter motivador e innovador en los procesos de enseñanzaaprendizaje. Debido a ello, cada vez son más los docentes que apuestan por dicha innovación metodológica (Armstrong y Landers, 2018; Buckley y Doyle, 2014), pues las características que definen el propio proceso de enseñanza-aprendizaje a través de la gamificación favorece la mejora de aspectos didácticos como: 1) la optimización del tiempo útil de clase; 2) el rendimiento del alumnado; 3) la motivación; 4) la ubicuidad del aprendizaje; 5) la satisfacción profesional del docente. Dentro del área de Ciencias Sociales, existen diferentes propuestas que han implementado la gamificación para el aprendizaje de la Historia en Educación Secundaria (Díaz et al., 2018; Gómez, 2018), donde las Tecnologías de la Información y la Comunicación (TIC), enriquecen el acto educativo y la adquisición de aprendizajes en un proceso conjunto y bidireccional (profesor-alumnado) de construcción del conocimiento respecto a la asignatura de Historia (Van Vaerenewyck et al., 2017; Voet y De Wever, 2017). 
Teniendo en cuenta todo ello, este estudio sitúa el foco en el análisis de la evaluación gamificada a través de la percepción del profesorado. Para ello, se diseña una situación experimental en la que el grupo control realizará un examen tradicional, mientras que, para el grupo experimental, la prueba de evaluación se transforma en un juego, comparando así ambas tipologías de examen desde la perspectiva docente. Con el fin de evitar el impacto de variables extrañas, la prueba se realizará sobre un mismo contenido del Curriculum de la asignatura de Historia en Educación Secundaria en centros educativos públicos de la misma comunidad autónoma durante el curso escolar 2018-2019. Además, respecto al grupo experimental, se le facilitó al profesorado objeto de estudio un modelo de examen gamificado similar. El motivo fue reducir diferencias entre los distintos grupos-clases que hicieran las pruebas, evitando que factores como la dotación de recursos tecnológicos del aula, la ratio de clase o la estructura curricular de la materia determinarán la valoración del profesorado. Partiendo de todo ello, los objetivos propuestos para este estudio son los siguientes: 1) conocer la percepción de los docentes sobre ambos tipos de prueba de evaluación; 2) comprobar si existen diferencias estadísticamente significativas en las percepciones de los docentes entre el examen tradicional y el gamificado. Bajo esta premisa, se establece la siguiente hipótesis estadística: considerando las percepciones de los docentes, se encontrarán diferencias significativas a favor del modelo examen gamificado respecto al tradicional.

\section{METODOLOGÍA}

La metodología se estructura en cuatro subapartados: enfoque metodológico, donde se explica la perspectiva desde la que se abordará el estudio; muestra, en la que se presentan los participantes y las principales características de los mismos; instrumento, detallando las características psicométricas del cuestionario y el contenido sobre el que versa; procedimiento, especificando las fases de implementación del proyecto y de recogida de la información; análisis de datos, donde se describen las diferentes pruebas estadísticas aplicadas.

\section{Enfoque}

Desde la perspectiva metodológica, se presenta un estudio cuantitativo de carácter descriptivo e inferencial con un diseño no experimental, recogiendo la información mediante un instrumento estandarizado.

\section{Muestra}

El muestreo se ha realizado mediante un procedimiento de carácter no probabilístico, partiendo de una selección intencionada de los docentes que han decidido participar. La población la forman docentes de Educación Secundaria que imparten la asignatura de Geografía e Historia en centros educativos de la provincia de Málaga (España). Para acceder a la información de dicha población, se recurrió a la Unidad Estadística de la Consejería de Educación de la Junta de Andalucía. Teniendo como punto de partida los datos facilitados por dicha administración educativa, se remitió a través del correo electrónico corporativo de los centros educativos una invitación formal para tomar parte en la investigación. Tras concretar la naturaleza de la propuesta, se solicitó la colaboración voluntaria de los docentes, generando un grupo control por aquellos que utilizarían el examen tradicional y un grupo experimental con aquellos que se prestaron a gamificar la prueba de evaluación siguiendo las indicaciones del equipo de investigación. De esta manera, la muestra final está compuesta por un total de 192 docentes de Geografía e Historia ( $n=192)$ durante el curso escolar 2018/2019, de los que 110 formaron el grupo control $(57.3 \% \%)$ y 82 el grupo experimental $(42.7 \%)$.

\section{Instrumento}

La recogida de información se ha realizado mediante la aplicación de un cuestionario estandarizado (tabla 1) sobre evaluación gamificada (Sánchez et al., 2019). Se trata de un cuestionario de 20 ítems categorizados en 5 dimensiones (aprovechamiento de clase; motivación; rendimiento; ubicuidad; satisfacción), donde los docentes deben manifestar su opinión respecto a los ítems formulados puntuando, mediante una escala de valor con formato tipo Likert con 5 opciones ( 5 = Siempre; 4 = Con frecuencia; 3 = A veces; 2 = Poco; 1 = Nunca), en función de la frecuencia con la que se cumplió el ítem en el modelo de examen que tuvo que realizar (grupo control el examen tradicional y grupo experimental el examen gamificado). Los valores alcanzados en cada ítem y dimensión fueron resultado de obtener las puntuaciones medias de las respuestas de los docentes de cada uno de los grupos.

Tabla 1: Estructura del cuestionario.

\begin{tabular}{|l|c|c|}
\hline Área de interés & Ítems & Variable dependiente (VD) \\
\hline Aprovechamiento de la sesión de evaluación & $1-4$ & 1 \\
\hline Motivación del alumnado frente a la prueba de evaluación & $5-8$ & 2 \\
\hline Rendimiento del alumnado en la prueba de evaluación & $9-12$ & 3 \\
\hline Continuidad del aprendizaje fuera del aula (Ubicuidad) & $13-16$ & 4 \\
\hline Satisfacción profesional del profesorado & $17-20$ & 5 \\
\hline
\end{tabular}


Atendiendo a los criterios de calidad del instrumento, se consideraron los parámetros de validez del contenido y de fiabilidad. En cuanto al proceso de validez del contenido, el cuestionario fue diseñado mediante un grupo de discusión, entre cuyas funciones estuvo la toma de decisiones respecto a las categorías a incorporar, el número de ítems o la escala de valoración, y validado por juicio de experto para ajustar el instrumento. La fiabilidad alcanzada, con una muestra de estudio de 217 participantes, fue de 0,914, lo que denota la bondad de la consideración interna del instrumento. En nuestro caso, la aplicación del coeficiente de Alfa de Cronbach reportó un valor de 0.865 , reflejando una fiabilidad aceptable. Es preciso subrayar que el instrumento fue aplicado del mismo modo que en la investigación de Sánchez et al. (2019) al implementarse en la misma zona y con solo un año de diferencia. Pese a que cambiaba la materia de los docentes y la etapa educativa, esto no supuso la necesidad de readaptar el instrumento, ya que el foco del estudio se centró en el modelo de examen y los factores relacionados con su desarrollo, siguiendo las mismas pautas para diseñar y aplicar los exámenes.

\section{Procedimiento}

El estudio se desarrolló en diferentes centros escolares durante el curso escolar 2018/2019. El instrumento se digitalizó y se administró a través de la aplicación Google Forms (online), debido a su flexibilidad, comodidad y gratuidad para la recogida de la información. El enlace a la URL se facilitó a los participantes a través de correo electrónico. Partiendo de que el instrumento es el mismo para ambos grupos, el proceso fue diferenciado. El grupo control recibió el cuestionario con las indicaciones para su cumplimentación tras la realización de un examen de Historia tradicional, el cual debía estar compuesto por preguntas tipo test o de desarrollo y presentarse en formato escrito. Por su parte, el grupo experimental requirió instrucciones para gamificar el contenido del examen, debiendo considerar pautas tanto para el diseño (tabla 2) como para la aplicación de la prueba de evaluación (tabla 3). Tras el diseño y aplicación de la prueba, los docentes del grupo experimental cumplimentan el cuestionario facilitado.

Tabla 2: Pautas para el diseño del examen gamificado. (Adaptado de Sánchez et al., 2019)

\begin{tabular}{|l|l|}
\hline Componente & Gamificación \\
\hline Denominación & Cambiar el concepto. Ya no hacen un examen, ahora juegan al Trivial \\
\hline Presentación & $\begin{array}{l}\text { Se presenta como un concurso, eliminando las connotaciones negativas asociadas a los exámenes. } \\
\text { El fin es reducir la tensión que se genera ante el examen tradicional }\end{array}$ \\
\hline Normas & $\begin{array}{l}\text { Modificar la presentación de las reglas. No se puede copiar o realizar la prueba en grupo, al igual que } \\
\text { en el examen tradicional, pero es el estímulo del propio formato el que genera que el alumnado } \\
\text { cumple las normas, y no por temor o imposición docente. }\end{array}$ \\
\hline Soporte & $\begin{array}{l}\text { La presentación de las preguntas se realizará de forma virtual, mediante una presentación } \\
\text { informática, en lugar de en papel. Las preguntas se proyectarán en las diapositivas, incorporando el } \\
\text { material que sea preciso (imágenes, vídeos, etc.) }\end{array}$ \\
\hline Premio & $\begin{array}{l}\text { El componente lúdico del examen gamificado consiste en lograr, durante la realización del examen, } \\
\text { responder el mayor número posible de respuestas de forma correcta, contabilizándose cada acierto } \\
\text { como un punto para su cuanta particular y obteniéndose el resultado final de la sumatoria total de } \\
\text { dichos aciertos. }\end{array}$ \\
\hline Comprobación & $\begin{array}{l}\text { Se concibe una gratificación a modo de regalo para el estudiante que alcance la puntuación más alta, } \\
\text { junto con subrayar su logro a nivel informativo para su reconocimiento público (a través del blog de } \\
\text { clase o el tablón de anuncios) }\end{array}$ \\
$\begin{array}{l}\text { La evaluación se hará entre todos. Cada alumno evalúa la prueba de otro compañero de clase } \\
\text { atendiendo a las explicaciones del docente sobre el contenido de cada pregunta. Con esta } \\
\text { explicación, se le concede al Trivial un valor añadido al convertirlo en una actividad más educativa } \\
\text { que examinadora. }\end{array}$ \\
\hline
\end{tabular}

Tabla 3: Pautas para la aplicación del examen gamificado. (Adaptado de Sánchez et al., 2019)

\begin{tabular}{|l|l|}
\hline Fase & Acción \\
\hline Preparación & $\begin{array}{l}\text { Docente y alumnado preparan, conjuntamente, una batería de preguntas tipo test (se puede realizar } \\
\text { mediante un documento colaborativo tipo Google Drive). Tras el diseño, el docente selecciona 15 } \\
\text { cuestiones y diseña una presentación informática ubicando, en cada diapositiva, una cuestión y las } \\
\text { opciones de respuesta. }\end{array}$ \\
\hline Presentación & $\begin{array}{l}\text { Se recomienda explicar las normas del Trivial en la primera diapositiva, entre las que encontramos: } \\
\text { 1) Responder a la pregunta que se formula por escrito y dentro del tiempo establecido (30 segundos); } \\
\text { 2) Está prohibido copiar; 3) Hablar durante el juego del Trivial resta puntos, descontándose al cómputo } \\
\text { de aciertos. }\end{array}$ \\
\hline
\end{tabular}


Tabla 3 (continuación)

\begin{tabular}{|l|l|}
\hline Fase & Acción \\
\hline Desarrollo & $\begin{array}{l}\text { Tras aclarar las normas, comienza la proyección de las diapositivas con las preguntas. El alumnado } \\
\text { deberá anotar sus respuestas en la libreta, con el fin de tener evidencias que pueda considerar el } \\
\text { docente para la evaluación continua. }\end{array}$ \\
\hline Finalización & $\begin{array}{l}\text { Tras concluir la presentación, los estudiantes se intercambian las libretas para llevar a cabo el proceso } \\
\text { de corrección. Durante el proceso, el docente reforzará los contenidos abordados en cada una de las } \\
\text { preguntas formuladas. Las respuestas correctas suman un punto. Se puede establecer un único } \\
\text { ganador (puntuación individual) o un equipo ganador (se agrupan estudiantes y se suman sus } \\
\text { puntuaciones) }\end{array}$ \\
\hline
\end{tabular}

\section{Análisis de datos}

Una vez recopilada la información de ambos grupos, se realizó el análisis de los datos mediante el paquete estadístico SPSS v.25. Teniendo en cuenta los objetivos propuestos, se realizaron diferentes pruebas. En una fase inicial, se obtuvieron los principales estadísticos descriptivos del conjunto de respuestas del instrumento, agrupando las puntuaciones en función del grupo de referencia, facilitando así el contraste entre la percepción de los docentes sobre cada tipo de examen en función de los ítems. Tras ello, se realizó también un análisis exploratorio de los estadísticos descriptivos de los dos tipos de prueba teniendo en cuenta las dimensiones que son consideradas en el instrumento. En una segunda fase, se analizó si existían diferencias significativas entre la prueba gamificada y la tradicional. Comenzamos por la comprobación de los supuestos paramétricos de homocedasticidad y normalidad. A este respecto, reflejar como el estadístico de Levene obtuvo una significatividad superior a 0.05 ( $p \geq 0.05$ ) en todas las variables salvo en la de motivación (VD2), siendo el valor de la homocedasticidad de las otras cuatro variables dependientes compatibles con la aplicación de pruebas paramétricas.

Tabla 4: Resultados de los supuestos paramétricos.

\begin{tabular}{|c|c|c|c|c|c|}
\hline \multirow[t]{2}{*}{ Área de interés } & \multirow[t]{2}{*}{ Tipo de examen } & \multicolumn{2}{|l|}{ Levene } & \multicolumn{2}{|c|}{ Kolmogorov-Smirnov } \\
\hline & & Estadístico & Sig. & Estadístico & Sig. \\
\hline \multirow{2}{*}{$\begin{array}{l}\text { Aprovechamiento de clase } \\
\text { (VD1) }\end{array}$} & Tradicional & \multirow[t]{2}{*}{3.533} & \multirow[t]{2}{*}{0.062} & 0.096 & 0.014 \\
\hline & Gamificado & & & 0.124 & 0.003 \\
\hline \multirow{2}{*}{$\begin{array}{l}\text { Motivación } \\
\text { (VD2) }\end{array}$} & Tradicional & \multirow[t]{2}{*}{16.451} & \multirow[t]{2}{*}{0.000} & 0.168 & 0.000 \\
\hline & Gamificado & & & 0.149 & 0.000 \\
\hline \multirow{2}{*}{$\begin{array}{l}\text { Rendimiento } \\
\text { (VD3) }\end{array}$} & Tradicional & \multirow[t]{2}{*}{1.805} & \multirow[t]{2}{*}{0.181} & 0.186 & 0.000 \\
\hline & Gamificado & & & 0.130 & 0.002 \\
\hline \multirow{2}{*}{$\begin{array}{l}\text { Ubicuidad } \\
\text { (VD4) }\end{array}$} & Tradicional & \multirow[t]{2}{*}{0.354} & \multirow[t]{2}{*}{0.552} & 0.187 & 0.000 \\
\hline & Gamificado & & & 0.153 & 0.000 \\
\hline \multirow{2}{*}{$\begin{array}{l}\text { Satisfacción } \\
\text { (VD5) }\end{array}$} & Tradicional & \multirow[t]{2}{*}{0.120} & \multirow[t]{2}{*}{0.729} & 0.122 & 0.000 \\
\hline & Gamificado & & & 0.128 & 0.002 \\
\hline
\end{tabular}

En cuanto a la normalidad, la prueba de Kolmogorov-Smirnov (con corrección de significatividad de Lillierfors), el nivel de significación es inferior a 0.05 ( $p \leq 0.05$ ), no ajustándose a la normalidad. Esta situación hizo que se descartaran las pruebas de carácter paramétrico y se tuviera que recurrir a las de carácter no paramétrico. La prueba elegida fue $U$ de Mann-Whitney. El motivo fue que nuestra intención era comparar medias de muestras independientes, pues los docentes que formaron parte de la muestra pertenecían a dos grupos diferentes (examen tradicional y gamificado) y se les cuestionó en una única ocasión (post intervención) sobre sus percepciones respecto al uso del examen tradicional (grupo control) y el gamificado (grupo experimental).

\section{RESULTADOS Y DISCUSIÓN}

Se presenta una selección de los resultados obtenidos en este trabajo. Comenzamos por el análisis descriptivo de las puntuaciones medias de los docentes (grupo control con examen tradicional y grupo experimental con examen gamificado) considerando su percepción sobre los ítems que componen el cuestionario y la escala para valorar la frecuencia de los mismos, de tipo Likert ( $5=$ Siempre; $4=$ Con frecuencia; $3=$ A veces; $2=$ Poco; $1=$ Nunca), utilizada . 
Tabla 5: Estadísticos descriptivos por tipo de prueba de evaluación.

\begin{tabular}{|c|c|c|c|c|c|}
\hline \multirow[t]{2}{*}{ Ítem } & & \multicolumn{2}{|c|}{ Examen tradicional } & \multicolumn{2}{|c|}{ Examen gamificado } \\
\hline & & Media & Desviación & Media & Desviación \\
\hline \multicolumn{6}{|c|}{ Aprovechamiento de la sesión de evaluación (VD1) } \\
\hline 1 & Aprendizaje durante la realización del examen & 2.03 & 1.15 & 3.51 & 0.84 \\
\hline 2 & Manifestación disruptiva escasa durante el examen & 3.68 & 1.23 & 3.72 & 1.25 \\
\hline 3 & Buen nivel de atención durante el examen & 3.55 & 0.93 & 3.48 & 1.03 \\
\hline 4 & El alumnado completa todas las tareas previstas & 3.31 & 0.92 & 4.00 & 0.92 \\
\hline \multicolumn{6}{|c|}{ Motivación del alumnado frente a la prueba de evaluación (VD2) } \\
\hline 5 & El alumnado manifiesta calma hacia la prueba de evaluación & 1.34 & 0.49 & 4.07 & 0.73 \\
\hline 6 & El alumnado adopta un papel activo en la tarea & 1.63 & 0.63 & 4.17 & 0.83 \\
\hline 7 & El alumnado manifiesta interés hacia la prueba de evaluación & 2.24 & 0.54 & 4.10 & 0.96 \\
\hline 8 & El alumnado manifiesta que el proceso de evaluación fue ameno & 1.31 & 0.48 & 4.55 & 0.69 \\
\hline \multicolumn{6}{|c|}{ Rendimiento del alumnado en la prueba de evaluación (VD3) } \\
\hline 9 & El alumnado aplica los conocimientos adquiridos con éxito & 3.41 & 1.10 & 3.41 & 0.68 \\
\hline 10 & El alumnado encuentra soluciones a los problemas planteados & 3.71 & 0.95 & 3.71 & 0.53 \\
\hline 11 & El resultado del aprendizaje es proporcional al esfuerzo & 3.35 & 0.82 & 3.37 & 0.78 \\
\hline 12 & Favorece el desarrollo de competencias claves & 2.79 & 0.79 & 4.06 & 1.16 \\
\hline \multicolumn{6}{|c|}{ Continuidad del aprendizaje fuera del aula (Ubicuidad) (VD4) } \\
\hline 13 & El alumnado realice la prueba de evaluación en casa & 1.55 & 0.67 & 4.04 & 0.79 \\
\hline 14 & El alumnado maneje la prueba de evaluación de forma autónoma & 1.71 & 0.68 & 4.11 & 1.05 \\
\hline 15 & El alumnado muestra la prueba de evaluación a su familia & 3.98 & 0.79 & 4.02 & 0.88 \\
\hline 16 & El alumnado muestra la prueba de evaluación a su grupo de pares & 2.51 & 0.82 & 4.34 & 0.89 \\
\hline \multicolumn{6}{|c|}{ Satisfacción profesional del profesorado (VD5) } \\
\hline 17 & El proceso de evaluación haya sido fructífero para el alumnado & 1.47 & 0.60 & 3.37 & 1.11 \\
\hline 18 & El proceso de evaluación sea coherente con la innovación pedagógica & 3.52 & 0.99 & 3.44 & 1.17 \\
\hline 19 & La sesión de evaluación concluyó de forma satisfactoria & 3.50 & 1.05 & 3.51 & 1.11 \\
\hline 20 & La intervención docente haya sido de calidad & 3.37 & 1.07 & 3.43 & 1.01 \\
\hline
\end{tabular}

A nivel general, la comparativa de las puntuaciones medias (tabla 1) subraya una mejor valoración del examen gamificado que del tradicional, siendo en las áreas de motivación y ubicuidad donde los ítems presentan más diferencia entre ambos tipos de evaluación. No obstante, esta diferencia no se registra en el ítem 15 "el alumnado muestra la prueba de evaluación a su familia" (área de ubicuidad), ya que se trata de un requerimiento impulsado desde la esfera educativa al solicitar, en muchos casos, la firma del propio examen por las familias para constatar que son conocedores de los resultados y la evolución del discente en este tipo de pruebas de evaluación.

Además de estas dos áreas de interés, destaca la diferencia en la opinión de los docentes en favor del examen gamificado respecto al tradicional en los siguientes ítems: "aprendizaje durante la realización del examen" (área de aprovechamiento de la sesión de evaluación), ya que la prueba tradicional está más orientada a la demostración de evidencias respecto a los contenidos fijados, mientras que el gamificado tiene una orientación más formativa que favorece la adquisición de nuevos aprendizajes, implicando al alumnado de forma activa tanto en el proceso de realización como de corrección; "favorece el desarrollo de competencias claves" (área de rendimiento del alumnado), donde la apuesta por una estrategia didáctica gamificada permite no solo adquirir contenidos sino también competencias necesarias en el ámbito histórico, coincidiendo con el estudio de Cózar-Gutiérrez y Sáez-López (2016), donde el 97\% del alumnado destacaba las ventajas de adquirir y desarrollar competencias en procesos de aprendizaje gamificados; y en el ítem "el proceso de evaluación haya sido fructífero para el alumnado" (área de satisfacción profesional del profesorado), donde los docentes reflejan una valoración positiva respecto al aprendizaje de sus discentes al sustituir el examen tradicional por el gamificado.

Por el contrario, en líneas generales, en las áreas de aprovechamiento de la sesión, rendimiento del alumnado y satisfacción profesional del profesorado, las diferencias entre ambos tipos de evaluación por ítems han sido menores, encontrando casos en los que la percepción del examen tradicional supera al gamificado (ítems 3 y 18) y otros en los que puntúan con igual valor (ítems 9 y 10), lo que nos permite tomar conciencia de los aspectos y factores que se deben considerar a la hora de plantear y diseñar este tipo de pruebas. Así, el examen tradicional fue percibido con mejor nivel de atención durante su realización, quedando reflejado en la tabla 5, donde la valoración del profesorado sobre el ítem 3 en el examen tradicional es de 3.55 puntos frente a los 3.48 puntos del examen gamificado, existiendo una diferencia de 0.07 puntos. Esto se debió a que el 
carácter lúdico del gamificado puede generar momentos de distracción puntuales durante su ejecución, mientras que el tradicional presenta menos estímulos en su diseño, junto con el establecimiento de normas y exigencias más estrictas que favorecen la menor pérdida de tiempo por parte de los discentes.

También, es relevante denotar como los participantes opinan que el examen tradicional es más coherente con la innovación pedagógica que el gamificado. Se trata de una circunstancia que, pese a su contradicción, acontece también en el estudio de Alfageme et al (2015), donde el profesorado no concibe la necesidad de cambiar la forma de evaluar tradicional mediante exámenes por el hecho de cambiar la práctica docente 0 llevar a cabo procesos de enseñanza-aprendizaje más innovadores. Manteniendo el foco en el plano descriptivo, es preciso ahondar en las diferencias existentes entre las percepciones sobre el examen tradicional y el gamificado a partir de las áreas de interés analizadas. Para calcular el valor de cada variable dependiente, se realizó la media de los cuatro ítems que conformaban el área de interés, considerando el valor medio obtenido por cada ítem en función del tipo de evaluación (figura 1).

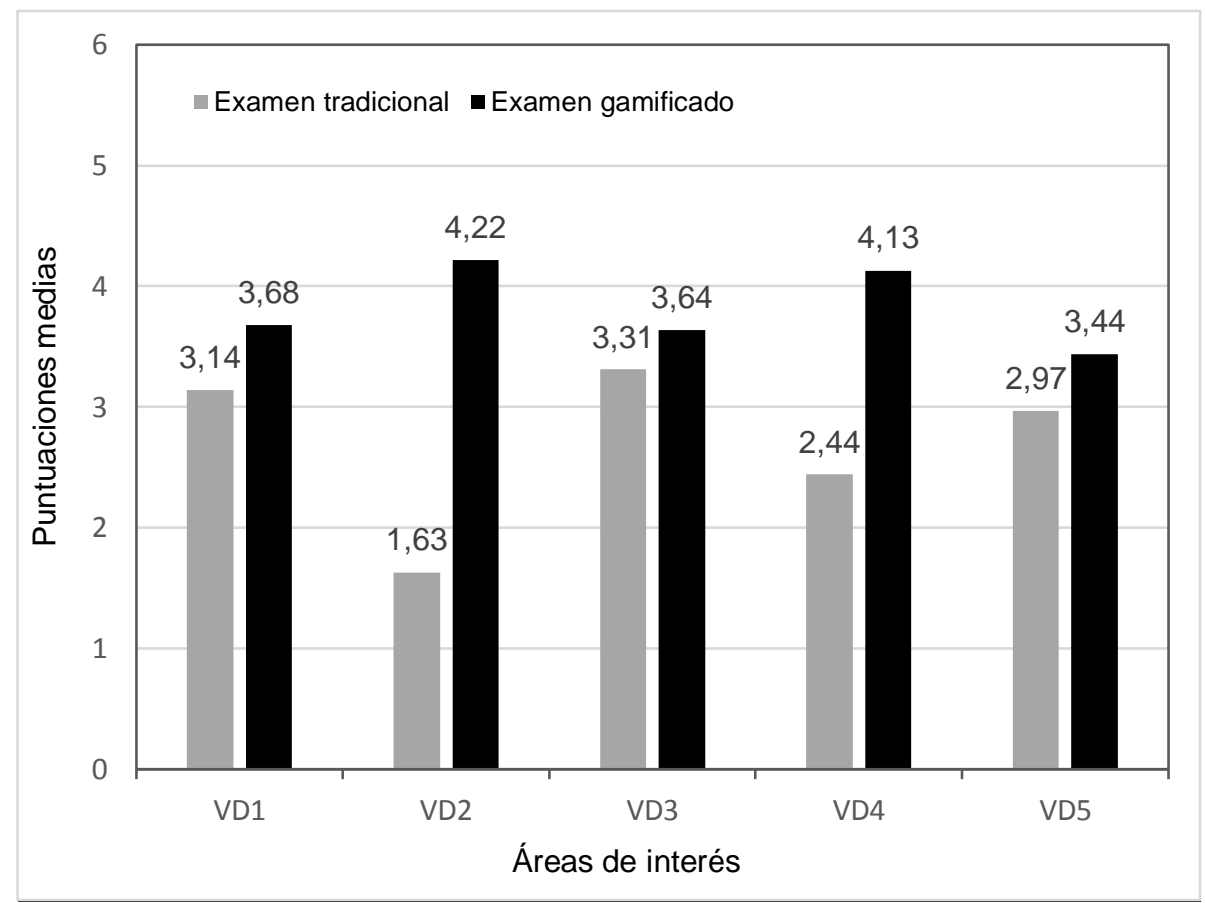

Fig. 1: Puntuaciones medias de cada variable dependiente en función del examen tradicional y el examen gamificado

Como se puede observar en la figura 1, en las áreas de interés vinculadas al aprovechamiento de la sesión de evaluación (VD1, 0.54 puntos de diferencia en las medias en favor del examen gamificado), el rendimiento del alumnado (VD3, 0.33 puntos de diferencia a favor del gamificado) y la satisfacción del profesorado (VD5, 0.47 puntos de media superior en el gamificado), las diferencias observables no son tan destacadas tras la aplicación del examen tradicional y el gamificado como en la variable motivación (VD2, 2.59 puntos de diferencia a favor del gamificado) y ubicuidad (VD4, 1.69 puntos de media superior en el gamificado).

Ante una sesión de evaluación con la misma duración y en la que, por su finalidad y propósito, las manifestaciones disruptivas suelen ser poco frecuentes, los docentes valoran que con el examen gamificado sacan más provecho (VD1) a dicha sesión que con el tradicional, no siendo amplía la diferencia entre ambos modelos (3.14 examen tradicional frente a 3.68 examen gamificado).

En lo que afecta a la variable de motivación (VD2), encontramos la mayor diferencia entre las puntuaciones de ambos tipos de prueba (1.63 examen tradicional, 4.22 examen gamificado). En los motivos, destacar que el examen gamificado sitúa el foco en la parte lúdica del aprendizaje, mientras que el tradicional está más orientado a la demostración del grado de adquisición de los conocimientos, focalizando en la capacidad memorística de los estudiantes (Bustos y Parra, 2019). Estos resultados (como apreciamos en la figura 1, la diferencia en favor del examen gamificado respecto al tradicional es de 2.59 puntos en la variable motivación) coinciden con diferentes estudios (Buckley y Doyle, 2014), en los que la motivación destaca como la principal ventaja de las propuestas didácticas gamificadas.

En la variable rendimiento (VD3) encontramos la menor diferencia respecto a los resultados obtenidos por ambos tipos de pruebas (3.31 examen tradicional frente a 3.64 examen gamificado), lo que refleja que una experiencia evaluativa lúdica puede mejorar la motivación, pero no impacta de forma tan notoria en los resultados alcanzados. Este hecho es contrario a los resultados de diferentes investigaciones (Riemer y Schrader, 2015; Su y Cheng, 2015) en las que si se alcanza mayor rendimiento con el uso de la gamificación 
respecto a propuestas más tradicionales. En este sentido, es preciso matizar que estamos midiendo resultados objetivos respecto al dominio de los contenidos mediante la realización de una prueba final, no ahondando en el proceso formativo ni las metodologías implementadas. De este modo, ante dos tipos de pruebas que ambas miden la eficiencia de los conocimientos del alumnado, es lógico que no se produzcan grandes diferencias en lo que a un tipo de evaluación sumativa (focalizada en el resultado final) se refiere, coincidiendo con el estudio de Sánchez et al. (2019). Además, también hay que considerar que se trataba de la primera experiencia del alumnado ante un examen gamificado en lugar de los convencionales, por lo que es necesario una adaptación a este tipo de pruebas para sacar un mayor aprovechamiento. Respecto a esto, sería interesante considerar, para futuros estudios, incluir participantes que ya hayan tenido experiencias con exámenes gamificados, con el fin de poder obtener un mayor rendimiento ante este tipo de pruebas.

Relacionado con la alta percepción de la motivación del examen gamificado está también el factor de la ubicuidad (VD4, obteniendo una diferencia en favor del examen gamificado respecto al tradicional de 1.69 puntos), ya que esta metodología de evaluación (formato concurso y disponibilidad de la prueba en el blog de clase) posibilita que el discente siga aprendiendo en casa a partir de los retos que el juego le plantea, incitando a que el alumnado vuelva a jugar (repetir la prueba) fuera del contexto escolar, al estar disponible el recurso. Si bien es cierto que no hay estudios que vinculen la gamificación de los exámenes de Historia con su impacto más allá del aula, el trabajo de Ojeda (2018) resalta la importancia del aprendizaje de la Historia para que el alumnado pueda entender la realidad con perspectiva crítica. En este sentido, el examen gamificado permite prolongar el proceso de aprendizaje más allá de la sesión de evaluación, debido al potencial que la gamificación juega en este proceso.

Respecto a la satisfacción docente (VD5), es preciso recordar que el profesorado refleja su valoración a tenor del desarrollo del proceso y de los resultados que se han obtenido con la prueba, no incorporando en dicha consideración la percepción que pueda tener el alumnado ante los diferentes tipos de evaluación. El situar el foco en la perspectiva docente se debe a la importancia de su papel para el desarrollo de procesos didácticos innovadores, de manera que un alto grado de satisfacción, como ocurre en nuestro estudio con la aplicación del examen gamificado, influirá positivamente en la mayor implantación de dichas propuestas. Este hecho coincide con investigaciones en las que los docentes reflejan encontrar recompensa profesional al implementar innovaciones pedagógicas exitosas en el contexto del aula, hecho también refutado con profesorado de Historia (Miralles et al., 2019a; Miralles et al., 2019b), del mismo modo que ocurre en nuestro trabajo. Por este motivo, para potenciar el uso positivo de esta innovación, es preciso mejorar la formación pedagógica de los docentes de Historia a este respecto, incorporando en la misma contenidos sobre la elaboración y diseño de procesos educativos gamificados.

Atendiendo a las diferencias que se han podido apreciar en el análisis descriptivo de las medias, realizaremos una comparación mediante estadística inferencial entre las puntuaciones obtenidas en las variables dependientes por cada tipo de prueba, teniendo como fin determinar si existen diferencias significativas entre las mismas. Para ello, aplicamos la prueba U de Mann-Whitney (tabla 6).

Tabla 6: Resultados del análisis factorial de la varianza (prueba U de Mann-Whitney).

\begin{tabular}{|c|c|c|c|c|c|}
\hline Área de interés & Tipo de examen & $\mathrm{N}$ & Media & Desviación típica & Significatividad \\
\hline \multirow[t]{2}{*}{ Aprovechamiento de clase } & Tradicional & 110 & 3.14 & 0.68 & \multirow{2}{*}{$.000^{*}$} \\
\hline & Gamificado & 82 & 3.68 & 0.57 & \\
\hline \multirow[t]{2}{*}{ Motivación } & Tradicional & 110 & 1.63 & 0.31 & \multirow{2}{*}{$.000^{*}$} \\
\hline & Gamificado & 82 & 4.22 & 0.47 & \\
\hline \multirow[t]{2}{*}{ Rendimiento } & Tradicional & 110 & 3.31 & 0.43 & \multirow{2}{*}{$.000^{*}$} \\
\hline & Gamificado & 82 & 3.64 & 0.45 & \\
\hline \multirow[t]{2}{*}{ Ubicuidad } & Tradicional & 110 & 2.44 & 0.37 & \multirow{2}{*}{$.000^{*}$} \\
\hline & Gamificado & 82 & 4.13 & 0.37 & \\
\hline \multirow[t]{2}{*}{ Satisfacción } & Tradicional & 110 & 2.97 & 0.59 & \multirow{2}{*}{$.000^{*}$} \\
\hline & Gamificado & 82 & 3.44 & 0.60 & \\
\hline
\end{tabular}

Por lo que respecta a los resultados, podemos observar la existencia de una significatividad estadística en todas las variables respecto a las diferencias entre ambos tipos de prueba de evaluación. Esto denota la valoración positiva del examen gamificado respecto al tradicional en los diferentes ámbitos que han sido considerados para el estudio. No obstante, como hemos comentado anteriormente, aunque a nivel estadístico se presenten diferencias significativas en todas las variables dependientes, la motivación (2.59 puntos de diferencia) y la 
ubicuidad (1.69 puntos de diferencia) han sido las áreas donde es mayor la distancia en las valoraciones dadas por el profesorado. Como podemos observar, los resultados denotan diferencias significativas en todas las áreas analizadas $(p \leq 0.05)$, siendo superior la percepción del profesorado respecto al examen gamificado respecto al tradicional en todas las variables consideradas (tabla 6). De este modo, una evaluación de tipo lúdica, dentro del ámbito didáctico de Historia, ofrece ventajas interesantes frente a una evaluación más tradicional, cuestión que no se refleja en diferentes estudios (Monteagudo y Vera, 2017) donde el examen memorístico sigue siendo el instrumento por excelencia.

Este hecho coincide con diferentes trabajos que subrayan los beneficios del uso de la gamificación como estrategia pedagógica (Domínguez et al., 2013; Landers y Amstrong, 2017), usando el juego para favorecer la adquisición de contenidos y mejorar la motivación respecto a la realización de tareas escolares. Así, partiendo de esta última idea, se considera interesante la posibilidad de gamificar no solo el proceso evaluativo sino también el de enseñanza. De este modo, se podrá trabajar en cambiar la concepción al respecto de que la innovación de los procesos formativos no tiene que ir vinculada a la modificación en la evaluación. Como hemos observado, el examen con base lúdica reporta mejoras en los criterios analizados respecto al proceso de aprendizaje, por lo que desarrollar las clases desde la gamificación podrá favorecer una mejora de los resultados en las áreas examinadas.

\section{CONCLUSIONES}

Considerando el análisis de los resultados alcanzados, la discusión de los mismos y su comparación con otros estudios e investigaciones, podemos extraer las siguientes conclusiones: 1) la evaluación gamificada ofrece ventajas respecto a la tradicional para la asignatura de Historia, siendo mejor valorada y percibida por los docentes, de forma general, respecto a las áreas de aprovechamiento de la sesión, la motivación del alumnado frente a la prueba de evaluación, el rendimiento del discente en el examen, la continuidad del aprendizaje fuera del aula (ubicuidad) y su propia satisfacción al implementar la prueba; 2 ) aspectos concretos como el nivel de atención durante la prueba de evaluación es mejor percibido por los docentes con el examen tradicional que con el gamificado, mientras que otros como la aplicación correcta de los conocimientos adquiridos o la solución a los problemas planteados, no presentan diferencias entre ambos tipos de evaluación; 3) debido a sus características, las áreas de motivación, vinculada al interés suscitado en el alumnado respecto al examen gamificado y la diversión que suscita su realización, y de ubicuidad, relacionada con la posibilidad de seguir aprendiendo de la prueba fuera del contexto del aula por su configuración (formato concurso) y por la temporalidad de la prueba tradicional (disponible en el blog de clase), reflejan las mayores diferencias entre las opiniones de los docentes respecto al examen tradicional y el de base lúdica; 4) a tenor de la valoración del docente sobre la satisfacción profesional que obtiene tras implementar la prueba gamificada, este modelo de examen se convierte en un recurso relevante para los procesos evaluativos, pudiendo ser una alternativa válida al examen tradicional.

\section{REFERENCIAS}

Acebedo, M.J., Aznar, I. e Hinojo, F.J., Instrumentos para la evaluación del aprendizaje basado en competencias: estudio de casos. dx.doi.org/10.4067/S0718-07642017000300012, Información tecnológica, 28(3), 107-118 (2017)

Agudelo, J.F., Rojas, F.S., Ocampo, E. y Clavijo, S.J., Sobre la evaluación escolar y su ética. dx.doi.org/10.4067/S071807642018000500071, Información tecnológica, 29(5), 71-80 (2018)

Alfageme, M.B., Miralles, P. y Monteagudo, J., ¿Cómo evalúa el profesorado de Geografía e Historia de Enseñanza Secundaria? doi.org/10.5209/rev_RCED.2015.v26.n3.44428, Revista Complutense de Educación, 26(3), 571-589 (2015)

Álvarez, E., La utilización de los nuevos contextos digitales como una herramienta alternativa para la enseñanza de la Historia. International Journal of New Education, 2, 95-113 (2018)

Armstrong, M.B. y Landers, R.N., Gamification of employee training and development. doi.org/10.1111/ijtd.12124, International Journal of Training and Development, 22(2), 162-169 (2018)

Bicen, H. y Kocakoyun, S., Perceptions of Students for Gamification Approach: Kahoot as a Case Study. doi.org/10.3991/ijet.v13i02.7467, International Journal of Emerging Technologies in Learning, 13(2), 72-93 (2018).

Buckley, P. y Doyle, E., Gamification and Student Motivation. doi.org/10.1080/10494820.2014.964263, Interactive Learning Environments, 24(6), 1162-1175 (2014)

Bustos, O.D. y Parra, K.L., Integración de las TIC en la enseñanza de la Historia en educación media superior. Revista boletín Redipe, 8(1), 106-113 (2019)

Colomo, E. y Domínguez, R., Definiendo identidades: el "canciograma" como herramienta metodológica de autoconocimiento, REICE, Revista Iberoamericana sobre Calidad, Eficacia y Cambio en Educación, 13(2), 131-146 (2015)

Cózar-Gutiérrez, R. y Sáez-López, J.M., Game-based learning and gamification in initial teacher training in the social sciences: an experiment with MinecraftEdu. https.//doi.org/10.1186/s41239-016-0003-4, International Journal of Educational Technology in Higher Education, 13(2), 1-11 (2016) 
Díaz, S., Diaz, J. y Arango, J., History Lessons on virtual worlds: How we can improve it? Campus Virtuales, 7(2), 81-91 (2018)

Domínguez, A., Saenz, J., y otros cuatro autores, Gamifying learning experiences: Practical implications and outcomes. doi.org/10.1016/j.compedu.2012.12.020, Computers \& Education, 63, 380-392 (2013)

Gómez, I.M., Gamification and technologies as innovative resources and strategies for the teaching and learning of history. doi.org/10.25053/redufor.v3i8.267, Educação \& Formação, 3(8), 3-16 (2018)

González, S.E., Cortés, J.A. y Lugo, N., Percepciones de docentes universitarios en el uso de plataformas tecnológicas gamificadas. Experiencias en un taller de formación. Innovación educativa, 19(80), 33-56 (2019)

Hanus, M.D. y Fox J., Assessing the effects of gamifcation in the classroom: A longitudinal study on intrinsic motivation, social comparison, satisfaction, effort, and academic performance. doi.org/10.1016/j.compedu.2014.08.019, Computer \& Education, 80, 152-161 (2015)

Landers, R.M. y Amstrong, M.B., Enhancing instructional outcomes with gamification: An empirical test of the TechnologyEnhanced training effectiveness model. doi.org/10.1016/j.chb.2015.07.031, Computers in Human Behavior, 71, 499-507 (2017)

Melo, D.S. y Díaz, P.A., El aprendizaje afectivo y la gamificación en escenarios de educación virtual. dx.doi.org/10.4067/S0718-07642018000300237, Información tecnológica, 23(3), 237-248 (2018)

Metzger, S.A y Paxton, R.J., Gaming History: a framework for what video games teach about the past. doi.org/10.1080/00933104.2016.1208596, Theory \& Research in Social Education, 44(4), $532-564$ (2016)

Miralles, P. y Monteagudo, J., Métodos, instrumentos y procedimientos para conocer cómo se evalúan las competencias históricas. dx.doi.org/10.1590/0104-4060.64404, Educar em revista, 35(74), 127-144 (2019)

Miralles, P., Gómez, C.J., Arias, V.B. y Fontal, O., Digital resources and didactic methodology in the initial training of History teachers. doi.org/10.3916/C61-2019-04, Comunicar, XXVII (61), 45-56, (2019a)

Miralles, P., Gómez, C.J. y Monteagudo, J., Perceptions on the use of ICT resources and mass-media for the teaching of History. A comparative study among future teachers of Spain-England. doi.org/10.5944/ educXX1.21377, Educación XX1, 22(2), 187-211 (2019b)

Monteagudo, J. y Vera, M.I., What Do Compulsory Secondary Education Students Learn about Art History? An Analysis of the Legislation and Exams in the Region of Murcia (Spain). dx.doi.org/10.6018/j/308981, Educatio Siglo XXI, 35(3), 229$254(2017)$

Monteagudo, J., Molina, S. y Miralles, P., Opiniones sobre evaluación de los profesores de segundo ciclo de ESO de Geografía e Historia en España, Revista Mexicana de Investigación Educativa, 66, 737-761 (2015)

Ojeda, R.M., Enseñanza de la historia en la educación superior a través de las TIC, Revista de la Universidad de La Salle, $75,105-127$ (2018)

Riemer, V. y Schrader, C., Learning with quizzes, simulations, and adventures: Students' attitudes, perceptions and intentions to learn with different types of serious games. doi.org/10.1016/j.compedu.2015.05.003, Computers \& Education, 88, 160-168 (2015)

Sánchez, E., Ruiz, J. y Sánchez, J., Gamification of Assessments in the Natural Sciences Subject in Primary Education. doi.org/10.12738/estp.2019.1.0296, Educational Sciences: theory \& practice, 19(1), 95-111 (2019)

Su, C. y Cheng, C., A mobile gamification learning system for improving the learning motivation and achievements. doi.org/10.1111/jcal.12088, Journal of Computer Assisted Learning, 31(3), 268-286 (2015)

Trevitt, C., Brenan, E. y Stocks, C., Assessment and learning: is it time to rethink student activities and academic roles? Revista de Investigación Educativa, 30(2), 253-269 (2012).

Van Vaerenewyck, L.M., Harlow, V. y Steckel, B., Sarah's Story: One Teacher's Enactment of TPACK+ in a History Classroom. Literacy research and instruction. doi.org/10.1080/19388071.2016.1269267, Literacy Research and Instruction, 56(2), 158-175 (2017)

Voet, M. y De Wever, B., Towards a differentiated and domain-specific view of educational technology: An exploratory study of history teachers' technology use. doi.org/10.1111/bjet.12493, British Journal of Educational Technology, 48(6), 1402-1413 (2017) 